\title{
LA CONSTRUCCIÓN DE UNA INDUSTRIA FARMACÉUTICA AUTOSUFICIENTE EN LA ESPAÑA DE LA AUTARQUÍA: ENTRE LA NECESIDAD, LA UTOPÍA Y LA PROPAGANDA FRANQUISTA*
}

\author{
Raúl Rodríguez Nozal \\ Área de Historia de la Ciencia \\ Unidad Docente de Salud Pública, Medicina Legal e Historia de la Ciencia \\ Departamento de Cirugía y Ciencias Médicas y Sociales \\ Universidad de Alcalá \\ Email: raulrn@uah.es \\ ORCID iD: http://orcid.org/0000-0003-2565-4695
}

Recibido: 2 febrero 2016; Aceptado: 25 septiembre 2016.

Cómo citar este artículo/Citation: Rodríguez Nozal, Raúl (2017), "La construcción de una industria farmacéutica autosuficiente en la España de la Autarquía: entre la necesidad, la utopía y la propaganda franquista", Asclepio, 69 (1): p173. doi: http://dx.doi. org/10.3989/asclepio.2017.05

RESUMEN: La historia de la industria farmacéutica española está condicionada por su adscripción a un modelo de corte mediterráneo, de menor empaque económico y mayor presencia de la componente artesana y profesional. La industria de las materias primas, al menos las de carácter químico-orgánico y fermentativo, no apareció hasta la dictadura franquista. Durante la Autarquía parece evidenciarse un cierto interés por promocionar este sector; en primer lugar, potenciando las industrias de productos naturales, algo que ya se venía haciendo con anterioridad a la Guerra Civil, con el propósito de obtener principios activos de acción medicinal y evitar así su importación; en segundo lugar, a más largo plazo, estableciendo en España una industria química integral, de tipo orgánico, capaz de obtener fármacos a partir del carbón; finalmente, tras conocerse la síntesis a escala industrial de la penicilina, entró un escena un nuevo objetivo que acabaría convirtiéndose en prioritario: la fabricación nacional de esta sustancia. Sin embargo hubo una serie de factores que frenaron el desarrollo de la industria químico-farmacéutica, como las propias limitaciones de las políticas autárquicas, el excesivo número de laboratorios y de productos comercializados, la escasa capitalización de estas empresas así como su tímida implicación en tareas investigadoras, y la insuficiente capacitación científico-técnica necesaria para esta actividad.

PALABRAS CLAVE: Industria químico-farmacéutica; Medicamentos industriales; Laboratorios farmacéuticos; Autarquía; Franquismo.

\section{THE DEVELOPMENT OF A SELF-SUFFICIENT PHARMACEUTICAL INDUSTRY IN AUTARCHIC SPAIN: BETWEEN NEED, UTOPIA AND FRANCOIST PROPAGANDA}

\begin{abstract}
The history of the Spanish pharmaceutical industry is conditioned by its ascription to a Mediterranean model, with small economic impact and a high presence of artisan and professional elements. The industry of organic and fermentative raw materials did not appear until the Franco dictatorship. During the autarchy period, an interest to stimulate the sector seems to have arose: first, by continuing the long going investment on industries of natural products to obtain medical active principles, hence preventing their import; secondly, aimed at long term, by establishing an integral organic chemistry industry to obtain drugs from coal; and finally, by promoting the development of an industry of penicillin synthesis. However, there were a number of elements that hindered the development of the chemical-pharmaceutical industry, including the inherent limitations of the autarchic policies, the excessive number of laboratories and commercialized products, the poor capitalization and lack of research programs of these companies and the insufficient technical and scientific abilities required for these activities.
\end{abstract}

KEY WORDS: Chemical-Pharmaceutical Industry; Industrial Drugs; Pharmaceutical Laboratories; Autarchy; Francoism.

Copyright: (C) 2017 CSIC. Este es un artículo de acceso abierto distribuido bajo los términos de la licencia Creative Commons Attribution (CC BY) España 3.0. 


\section{INTRODUCCIÓN}

Existe un cierto consenso entre los historiadores de la economía respecto de la consideración del franquismo como un período heterogéneo desde el punto de vista del desarrollo industrial; algunos autores dividen estos años de dictadura prácticamente por décadas, finalizando la Autarquía en 1950 ó 1951 (García Delgado, 1994; Martínez Segarra, 1987); otros identifican el período autárquico a la manera clásica, es decir, entre los años 1939 y 1959 (González González, 1979; Barciela (ed), 2003; Catalán, 2002); incluso hay quien opina que, aunque la Autarquía se vio reducida a partir de 1959 con el Plan de Estabilización, no desapareció totalmente hasta 1986, con el ingreso de España en la Comunidad Europea (Tamames Gómez, 2005).

Son muchos los autores que están convencidos de que las políticas autárquicas obstaculizaron el desarrollo económico e industrial de nuestro país (Fontana, 2004; Arco Blanco, 2006); Catalán, 1989; Fraile Balbín, 1991; Miranda Encarnación, 2003; Moreno Fonseret, 2002; Gómez Mendoza, 1997; Tamames Gómez, 2005). Los posicionamientos proteccionistas y de intervención estatal ya habían sido favorecidos años antes, durante la Dictadura de Primo de Rivera y, en cierto modo, fueron prorrogados en la II República; la Autarquía incrementó esta componente nacionalista (Sánchez Recio, 2003; Fernández Navarrete, 2005, p. 50) con medidas tendentes a la restricción de la libre circulación de mercancías y la inversión extranjera -con el consiguiente empobrecimiento tecnológico-, el control de los trabajadores, la planificación productiva sectorial, las políticas monetarias y energéticas de carácter restrictivo (Deu y Llonch, 2013, p. 19) o el apoyo "para favorecer los planes industrialistas de los militares" (López García y Cebrián Villar, 2015, p. 411).

Todos estos factores acabaron actuando como freno para el desarrollo de la industria químico-farmacéutica española durante la Autarquía, de la misma manera que fueron determinantes en otros sectores industriales, como la Agricultura (Fernández Prieto, 2007), la industria del calzado (Miranda Encarnación, 1994), la textil (Deu y Llonch, 2013) o la de la piel (Colomer i Roma, 2003), por poner sólo algunos ejemplos. Pero, además, existen otros factores ralentizadores, que iremos desarrollando a lo largo de este trabajo, que son característicos de la industria del medicamento, en gran medida derivados de su adscripción a un modelo de industrialización farmacéutica de corte mediterráneo (González Bueno y Rodríguez Nozal, 2010): atomización de los recursos en un elevado número de laboratorios y productos comercializados, escasa capitalización de estas industrias, a menudo empresas familiares, poca implicación en tareas investigadoras e insuficiente capacitación científico-técnica, necesaria para llevar a cabo estas actividades.

El análisis del proceso de industrialización farmacéutica en España con anterioridad a la Guerra Civil ha sido objeto de nuestro grupo investigador a través de varios proyectos de investigación (CAM 06/0072/98; BHA 2002-01580; y HUM2005-04505) y un buen número de publicaciones, entre las que destacan los libros titulados Entre el Arte y la Técnica. Los orígenes de la fabricación industrial del medicamento y La industrialización del medicamento en la España Contemporánea. Desde hace algunos años esta línea investigadora se sitúa en el franquismo (Proyectos HAR 2009-09564 y HAR 2013-42536P); en el texto titulado La Tutela Imperfecta. Biología y Farmacia en la España del primer franquismo ya se han plasmado algunos resultados de estas investigaciones, en concreto las centradas en el Cuerpo Militar de Farmacia, el Sindicato Vertical de Industrias Químicas como elemento gestor del proceso de industrialización farmacéutica, el medicamento veterinario, las patentes de sulfamidas durante este período, el estudio monográfico de algunos laboratorios españoles o ciertos aspectos relacionados con la producción de antibióticos durante el primer franquismo, asunto que ha sido objeto de otros trabajos de investigación publicados recientemente (Rodríguez Nozal, 2011b; González Bueno, Rodríguez Nozal y Pérez Teijón, 2012). El tema de los antibióticos también ha sido estudiado por otros grupos investigadores (Santesmases, 1999; Santesmases, 2001; Santesmases, 2010; Santesmases y Gradman -eds-, 2011; Romero de Pablos, 2011; Cozzoli, 2014).

Otros compañeros, en particular Nuria Puig Raposo, han estudiado algunas de las claves relacionadas con el funcionamiento de la industria químico-farmacéutica desde el punto de vista de la formación de redes empresariales (Puig Raposo, 2004), así como las grandes empresas químico-farmacéuticas de capital alemán instaladas en España y su proceso de nacionalización tras los acuerdos de Bretton Woods (Puig Raposo, 2003; Puig Raposo, 2002; Puig Raposo y Álvaro Moya, 2007).

Con todo, aún quedan muchos asuntos a los que prestar atención en lo que a la industria del medicamento durante el franquismo se refiere: el fenómeno del "exilio interior" que tuvieron que soportar algunos 
de nuestros más importantes científicos, a menudo recluidos en laboratorios farmacéuticos; la investigación sobre medicamentos desarrollada por la industria farmacéutica española; el estudio de las conexiones empresariales a través de diferentes laboratorios o el inventariado de todos los laboratorios y personas involucradas en la industria farmacéutica española durante el franquismo, asunto en el que actualmente estamos ocupados.

Otro de los aspectos que aún quedaban por tratar, y que constituye el principal objetivo de este trabajo, es el relativo a la producción de materias primas para la industria del medicamento. Teniendo en cuenta que este sector apenas tuvo presencia en nuestro país con anterioridad a la Guerra Civil, y que estas sustancias eran habitualmente importadas de países como Alemania o Suiza, nos interesaba conocer cómo trató de ser contrarrestada esta situación de dependencia exterior, que violentaba el espíritu de las políticas autárquicas imperantes a comienzos del franquismo; y si realmente nuestro gobierno estaba en disposición de hacer lo que fuera necesario para establecer en España una industria química capaz de autoabastecer las necesidades internas de materias primas con las que fabricar medicamentos; o, como cabría esperar, cuáles podrían haber sido las razones (más allá del finisecular atraso científicoindustrial en esta materia) que pudieran explicar el fracaso de este utópico planteamiento, construido desde la propaganda franquista. Asimismo, también nos interesaba conocer cuál fue la posición del gobierno ante las materias primas de origen natural: productos vegetales, sueros, vacunas y antibióticos, éstos últimos en plena expansión durante los años cuarenta y cincuenta.

Para la realización de este trabajo nos hemos valido de fuentes primarias impresas, algunas publicadas en prensa científica y profesional, como Farmacia Nueva, El Monitor de la Farmacia y de la Terapéutica, Circular Farmacéutica, Química e Industria, Información Comercial Española o Ion, el órgano oficial del Sindicato Vertical de Industrias Químicas. Otras son monografías escritas por algunos de los principales impulsores de las políticas químicas y farmacéuticas durante la Autarquía, así como por voces destacadas de la Administración, de la Farmacia y de la empresa privada, como Víctor Villanueva Vadillo, Ángel Rubio Carsi, Juan Abelló Pascual, Federico Mayor Domingo, Francisco Donada Bosch, Carlos Alfageme Rubio, José Amargós Anoro, Salvador Brosa Rabassa o Antonio Gallego Fernández.

\section{LA INDUSTRIA QUÍMICO-FARMACÉUTICA EN ESPAÑA CON ANTERIORIDAD A LA GUERRA CÍVIL}

En el medicamento industrial podemos distinguir dos componentes fundamentales: la sustancia con actividad terapéutica, generalmente de origen químico o extractivo; y el producto acabado, sometido a operaciones de tecnología galénica (compresión, encapsulación, etc.), envasado y etiquetado. Algunos países, sobre todo del ámbito centroeuropeo / protestante, se volcaron hacia la Química Orgánica de aplicación industrial; eran generalmente fábricas de colorantes artificiales, en las que también se obtenían explosivos, materiales fotográficos, perfumes y, por supuesto, medicamentos. No eran, como puede entenderse, laboratorios farmacéuticos en sentido estricto, sino grandes fábricas químicas altamente capitalizadas, con gran cantidad de mano de obra y de personal muy cualificado, y una separación clara entre la propiedad y la dirección técnica. En cambio, las naciones del entorno mediterráneo, de cultura católica, concebían la industria farmacéutica como una actividad independiente de la Química, de donde obtenía las materias primas con las que componer un producto generado por tecnología galénica y listo para el consumo; en estas pequeñas fábricas, a veces simples laboratorios anejos a farmacias, en las que sólo se hacían medicamentos, la propiedad y el equipo técnico solían recaer en las mismas personas, generalmente profesionales de la Farmacia ${ }^{1}$.

La historia de la industria farmacéutica española está condicionada por su adscripción a un modelo de corte mediterráneo, de menor empaque económico y mayor presencia de la componente artesana y profesional. Puede decirse que no hubo industria químico-farmacéutica en España, al menos de carácter químico-orgánico o fermentativo, hasta la dictadura franquista. En opinión del industrial J. Agell i Agell (1926), la fabricación de productos farmacéuticos tenía un gran desarrollo "en cuanto a formas y preparados y especialidades farmacéuticas" aunque no podría decirse lo mismo en cuanto a las "especies químicas, pues sobre todos orgánicos, son escasísimos los que aquí se producen". Es verdad que éramos capaces de producir una pequeña cantidad de residuos (alquitranes o breas) obtenidos a partir de la hulla blanca, la materia de partida para la obtención de medicamentos pero, o bien se consideraban residuos sin más y se eliminaban, o eran vendidos a empresas extranjeras de colorantes y explosivos. Sin embargo hubo algún intento, fallido, de obtener colorantes sintéticos con anterioridad a la Guerra Civil²; es el caso de de la bar- 
celonesa "Fabricación Nacional de Colorantes y Explosivos" aunque, tal y como ha manifestado Nùria Puig Raposo (1999, pp. 113-114 y 128), puede considerarse como "el fracaso de la primera empresa española moderna del sector".

Las circunstancias adversas, de carácter político, económico o sociológico, que padeció España a finales del siglo XIX y principios del XX, no fueron las más idóneas para el desarrollo industrial. Si a estos problemas añadimos otros, derivados de la propia incapacidad de nuestros gobernantes y de su arrogante suficiencia en materias que no dominaban, obtenemos un resultado de lo más desalentador. A las propias insuficiencias del tejido químico-farmacéutico y a la insuficiente implicación del Estado, habría que sumar el pobre estado en el que se encontraba la enseñanza universitaria en química aplicada (Rodríguez Nozal, 2004b).

A pesar de los intentos por desarrollar en España una industria químico-farmacéutica de base (Rodríguez Nozal, 2004b), en la que trataron de implicarse algunos políticos y figuras destacadas de la Farmacia, lo cierto es que la industria químico-farmacéutica anterior a la Guerra Civil se circunscribió al ámbito de los laboratorios de transformación de producciones naturales, un tejido fabril pobre, muy atrasado desde el punto de vista científico y tecnológico, poco dado a la exportación e incapaz de surtir de materias primas a los laboratorios de medicamentos industriales. ${ }^{3}$

Ya en plena Autarquía, algunas voces destacadas del ámbito químico y farmacéutico (Villanueva Vadillo; Chocano y Gil Collado, 1955) se mostraban francamente duras con las políticas industriales mantenidas con anterioridad a la Guerra Civil; pensaban que los gobiernos anteriores a Franco habían vivido "de espaldas al proceso de industrialización" y que no fueron capaces de crear una industria fuerte, viable y nacional; llegan a calificar a esta industria de "vigorosa en su planteamiento y concepción, lánguida en su desarrollo y pobre en su economía". Abundando en este análisis, una voz tan autorizada y comprometida con el Régimen, como la de Víctor Villanueva Vadillo (1948, p. 302), criticaba la política española anterior a Franco, tildándola de inconsciente, irresponsable e incrementadora del endeudamiento al no seguir el modelo trazado por otros países en lo relativo a la creación de "un programa nacional de ordenación económica, en el que la industria químico-farmacéutica hubiera podido tener un sólido contenido"; en su opinión, una política que sólo pudo ser posible gracias a nuestras grandes reservas de oro y a la "normalidad internacional", que permitía las importaciones aunque fueran gravosas para el Estado.

\section{ESTADO DE LA INDUSTRIA QUÍMICO-FARMACÉUTICA ESPAÑOLA DURANTE LA AUTARQUÍA: ENTRE LA AU- TOCAOMPLACENCIA Y LA UTOPÍA}

Estas duras opiniones hacia las políticas industriales pertenecen a uno de los principales actores de la industria farmacéutica española durante el franquismo, el farmacéutico burgalés Víctor Villanueva Vadillo. ${ }^{4}$ Él fue quien trató de apoyar la política en materia de industrias químico-farmacéuticas, durante la Autarquía, desde su privilegiada posición en el Sindicato Vertical de Industrias Químicas ${ }^{5}$, un organismo integrado en la Organización Sindical Española, definitivamente establecida a finales de 1940, a través de una Ley "de Bases de la Organización Sindical".

Una de las misiones encomendadas a este Sindicato Vertical fue la de controlar, regular y organizar todo lo relativo a las materias primas necesarias para el desarrollo de estas industrias; en opinión de algunos autores (Moreno Fonseret, 1999, pp. 100 y 108-109), "el poder que daba el reparto de los cupos de materias primas, energía o los pedidos oficiales convirtió a los Jefes Sindicales en omnipotentes gestores clandestinos, que repartieron con total impunidad dichas materias a su antojo o, en ocasiones, intervinieron directamente en el mercado negro". Las empresas pequeñas no lo debieron pasar muy bien, pues tuvieron que luchar contra estos oligopolios y contubernios entre empresarios y jefes sindicales ${ }^{6}$, además de asumir que, en el concepto global de la Organización Sindical, se preferían pocas industrias pero bien asentadas, que fueran capaces de asumir con eficacia los cupos de materias primas, evitando así que éstos quedaran excesivamente atomizados. Sea como fuere, una de las consecuencias inmediatas del nuevo orden sindical, sobre todo para aquellos laboratorios necesitados de materias primas (la mayoría), fue el encuadramiento obligatorio de trabajadores y empresarios dentro de un determinado servicio o rama de producción. ${ }^{7}$

La escasez de materias primas fue un freno para el desarrollo de la industria farmacéutica y, desde luego, preocupaba a nuestras autoridades como bien puede comprobarse en las actas de los grupos y subgrupos incluidos en el Sindicato Vertical de Industrias Químicas relacionados con el medicamento ("Productos Farmacéuticos", "Especialidades" y "Dietéticos"), publicadas en la revista Ion, órgano de expresión de este Sindicato; en ellas se informaba de la escasez de algunas materias primas (azúcar, alcohol, aceite, glicerina, etc.), así como de los mecanismos y sistemas de declaración obligatoria por las que deberían pasar los laboratorios si querían optar a los cupos. 
En este contexto de escasez de materias primas, el ingeniero industrial C. Abollado Aribau (1945) tan sólo señalaba dos industrias farmacéuticas españolas en las que se elaboraban también materias primas de uso medicinal, FAES y $A b e l l o^{8}$, el resto no eran más que centros en los que se envasaban medicamentos procedentes del extranjero o, a lo sumo, establecimientos fabriles de corte exclusivamente galénico. Si exceptuamos estos laboratorios farmacéuticos y alguno más, como Esteve, responsable de la primera síntesis química española del "neo-salvarsán" (el "neo-spirol") durante los primeros años de la década de 1930; Andreu, fabricante de productos sulfamídicos ya desde 1935; Laboratorios del Norte de Espa$\tilde{n} a$, especializado en preparados oftalmológicos; o los de tipo biológico, que mencionaremos un poco más adelante, la industria químico-farmacéutica y de las materias primas al comienzo de la Autarquía era una actividad poco relevante.

Sin embargo, durante estos años, parece evidenciarse un cierto interés por promocionar este sector a través de una política organizada en tres etapas; en primer lugar, potenciando las industrias de productos naturales, algo que ya se venía haciendo con anterioridad a la Guerra Civil, con el propósito de obtener principios activos de acción medicinal y evitar así su importación, es más, incluso favorecer su exportación; en segundo lugar, a más largo plazo, estableciendo en España una industria química integral, de tipo orgánico, capaz de obtener fármacos a partir del carbón, tal y como hacían las grandes potencias internacionales; finalmente, tras conocerse la síntesis a escala industrial de la penicilina, hacia 1944, entró un escena un nuevo objetivo que, en poco tiempo, acabó convirtiéndose en prioritario: la fabricación nacional de esta sustancia.

A pesar de las críticas vertidas por Víctor Villanueva hacia las políticas en materia químico-industrial mantenidas por los gobiernos anteriores a Franco, que señalábamos con anterioridad, lo cierto es que durante la década de los cuarenta se continuó en la inercia de años anteriores; es decir, la de favorecer las industrias de droguería medicinal dada la riqueza española en esta materia, habitualmente exportadora de plantas medicinales que, en otros países, eran transformadas en principios activos; de esta manera, volvían a entrar en nuestro país, con el consiguiente desequilibrio para nuestra balanza comercial, además de atentar contra las propias directrices autárquicas emanadas desde el gobierno de la nación. Para Víctor Villanueva (1945, pp. 123-124), esa industria era un "voluminoso y rico filón" suscep- tible de "industrialización inmediata", al contrario de lo que sucedía con la industria de base orgánica, aún necesitada de personal especializado, medios y tiempo para su establecimiento, aunque ésta debería constituir el nuevo campo de actuación de los farmacéuticos españoles, por donde deberían ir "racional y progresivamente canalizándose sus actividades". Con el fin de conseguir estos propósitos, Víctor Villanueva (1945, pp. 129-135 y 137-146) pone a dos industrias como ejemplos a seguir: la de la efedra y la del cornezuelo de centeno.

En cuanto a la industria química orgánica durante los primeros años de la Autarquía, la mayor parte era deudora del carbón, la principal materia prima en aquella época. Víctor Villanueva (1945, pp. 15-105) reconocía que no éramos siquiera capaces de mantener nuestras propias necesidades, lo "que imposibilita la realización del vasto programa industrial químico (...) Si el déficit de hulla se arrastra lógicamente en todos los productos a que conduce su destilación, donde se acusa más hondamente es en la materia prima acaso más fecunda de este sector químico: el alquitrán". Según datos de Villanueva, en España había, en 1943, cincuenta y dos fábricas de gas y nueve baterías de hornos de coque, donde se obtenía todo el alquitrán de hulla español; en total 36.015.645 kilos, más de la mitad obtenidos por Altos Hornos de Vizcaya y el $20 \%$ por Duro Felguera. Este alquitrán se destinaba a ferrocarriles (70\%), carreteras (18\%) e industrias varias (12\%). Por lo tanto, estamos ante un producto deficitario en España que, además, se empleaba en sectores estratégicos como los transportes (ferrocarriles y carreteras), los explosivos o el ejército.

Pocos años después, Víctor Villanueva (1948) volvía a realizar balance de la industria químico-farmacéutica española a través de un trabajo que, a juzgar por lo reiterado de su publicación ${ }^{9}$, debió de ser bien conocido por la comunidad farmacéutica, científica e, incluso, económica del país. En él ámbito de las industrias establecidas a partir de las sustancias naturales de origen vegetal, la rama donde más se desarrolló la industria químico-farmacéutica durante los años cuarenta, siguen a la cabeza las fábricas para la obtención de efedrina, principios activos del cornezuelo de centeno, a las que se unen otras como las de algas para la obtención de ácido algínico, alginatos y yodo, hoja de gayuba, jara para la extracción de láudano y aceites esenciales de uso en perfumería, digital, tabaco, scila maritima, belladona, "cilindro de arroz", adormidera, cascarilla de cacao, naranjas y limones, resinas, semillas de ricino o germen de trigo. 
Dentro de este grupo de industrias relativamente sencillas, de manipulación de materias primas de origen natural, también menciona otras fábricas concebidas a partir de sustancias de origen mineral, como la sal para la obtención de cloruro sódico puro, bromo, bromuro potásico y bromuro sódico; y de origen animal, como el suero de leche para la fabricación de lactosa farmacéutica, los productos opoterápicos -según Villanueva capaces de mantener el $80 \%$ de la demanda patria y con cifras que podrían ser mayores si no se desperdiciasen las glándulas de animales sacrificados en los mataderos-, y otras sustancias como pectina, albúmina, colesterina, lanolina, lecitina, etc. De las industrias químico inorgánicas apenas se ocupa, más que para decir que ya existían, al menos cuanto a la síntesis de productos como los ácidos clorhídrico, sulfúrico, nítrico o el amoniaco.

La lista de productos de síntesis de origen orgánico era más extensa aunque, salvo excepciones, no eran los más habitualmente utilizados en la fabricación de medicamentos. ${ }^{10}$ El químico Antonio Mora López (1951, p. 2023) calificaba de "triste panorama” el de la síntesis orgánica en España al finalizar la Guerra Civil aunque, en 1951, era optimista o, tal vez, patriotero en exceso al valorar de "cambio radical" la situación en este sector "en un sentido realmente favorable" por lo que consideraba que no parecía "aventurado predecir que dentro de muy pocos años su situación será francamente satisfactoria de persistir las circunstancias que han permitido esta saludable reacción" ${ }^{11}$ Por esas mismas fechas, la visión de Víctor Villanueva Vadillo (1952) era aún más optimista que la de Antonio Mora López, bien podría decirse que rayaba en la utopía propagandista ya que, a su entender, "solo razones de sensata coordinación limitan las ansias de su instintivo y veloz crecimiento. Programas perfectamente maduros esperan solo el turno para desarrollarse cuando las previsiones de este conjunto plan armónico lo aconsejen".

Pero no todas la opiniones eran tan favorables hacia las políticas autárquicas en lo que a la industria químico-farmacéutica se refiere; desde las páginas de EI Monitor de la Farmacia, "Un Colaborador" (1942) -ni siquiera se atrevía a firmar con su nombre- se mostraba escéptico con las bondades de este sistema económico, que se había mostrado ineficaz con este tipo de actividad al considerar que una industria químico-farmacéutica nunca podía ser totalmente independiente al estar ligada a otras, como la de los perfumes o las materias colorantes.
Donde probablemente más se progresó durante la década de los años cuarenta fue en el terreno de los productos biológicos, como los sueros, las vacunas y los antibióticos. En cuanto a estas primeras sustancias, ya se fabricaban con anterioridad a la Guerra Civil, en empresas tan conocidas como IBYS, Llorente, Instituto Bioquímico Hermes, FHER, THIRF o Instituto Ferrán, por mencionar sólo aquellos que registraron el mayor número de estos productos (Gomis, 2008). Víctor Villanueva Vadillo (1948, p. 309) no ocultaba su entusiasmo ante este sub-sector de la industria farmacéutica aunque, como acabamos de comentar, no parece que se debiera a las bondades de la Autarquía, sino a una situación consolidada con anterioridad a 1936.

La gran apuesta del primer franquismo en materia químico-farmacéutica fue el establecimiento en nuestro país de una industria propia de penicilina y otros antibióticos, ya que, en palabras de Víctor Villanueva (1948, p. 309), en el orden económico representaba "el renglón más grande de divisas que un solo medicamento nos reclama y en el sanitario, la constante pesadilla de la dependencia extranjera para nuestro aprovisionamiento". Era, efectivamente, un bien escaso que, desde 1944, entraba vía importación controlada y, más frecuentemente, se obtenía a través del mercado negro; en ambos casos, situaciones inaceptables para quienes habían planificado un concepto autárquico de la economía española.

Al contrario de lo que sucediera con las industrias de materias primas procedentes de la Química Orgánica, poco promocionadas o potenciadas por la política científica e industrial de la Autarquía, la producción al por mayor de sustancias antibióticas para la preparación de medicamentos sí contó con la complicidad de nuestros gobernantes. Un Decreto de 1-IX1948 (BOE, 6-X-1948) declaraba de 'interés nacional' la fabricación de penicilina y abría un concurso para designar las dos entidades españolas que habrían de monopolizar esta actividad. En 1949 se resolvía en favor de dos consorcios empresariales: Antibióticos y Compañía Española de Penicilina y Antibióticos (CEPA). Sin embargo, la producción nacional de penicilina nunca fue un proyecto totalmente autárquico, bien al contrario estuvo muy supeditado a la ciencia y la tecnología estadounidense; Antibióticos se valió de la colaboración y las patentes del laboratorio farmacéutico Schenley, mientras que CEPA hizo lo propio con la firma Merck. ${ }^{12}$ Algunos años después, en 1953, se concedió una nueva licencia nacional en favor del grupo empresarial Alter ${ }^{13}$, a través de su participada 
Farmabión; en esta ocasión, la colaboración científica y técnica no fue norteamericana sino danesa, a través del laboratorio danés Leo Pharmaceutical Products. ${ }^{14}$

La colaboración norteamericana en materia de antibióticos, visible desde finales de 1948 (Redondo Rincón y González Bueno, 2013, p. 262), probablemente se vio favorecida tras la adhesión del gobierno de Franco a los acuerdos de Bretton Woods que, en lo que respecta a España, modificó radicalmente el incipiente mapa químico industrial español, hasta entonces dominado por las empresas alemanas, algo especialmente notorio en las químico-farmacéuticas (Bayer, Merck, Schering, Böhringer, etc.) $)^{15}$; y al declive, tras la finalización de la II Guerra Mundial, del liderazgo germano basado en el carbón y en la química orgánica tintórea, que fue sustituido gradualmente por la hegemonía estadounidense fundamentada en la explotación industrial del petróleo y en la fabricación a gran escala de productos como las fibras sintéticas, el DDT y, por supuesto, los antibióticos (Puig Raposo, 2004).

Este fenómeno, conocido como "americanización en la economía española" ya se había consolidado en Europa hacia 1945. Sin embargo, en España se produjo de manera más tardía, en la década de los cincuenta y, aún de manera más evidente, durante los sesenta. ${ }^{16}$ En 1953 hizo su aparición un programa de ayuda económica para nuestro país equivalente al "Plan Marshall", aunque de menor cuantía económica; aún así favoreció las actividades estadounidenses en España y, también, las inversiones directas. Lina Gálvez Muñoz y Francisco Comín Comín (2003, p. 141) califican al período 1939-1959 como la "etapa de mayor hostilidad a la empresa extranjera", aunque reconocen que éstas "no dejaron de estar presentes en el país debido a la dependencia tecnológica que tenía la economía española". De acuerdo con esta visión, la participación estadounidense en el programa nacional de producción de antibióticos, ejecutada en fecha muy temprana (1949), tal vez deba considerarse como un claro antecedente de este proceso de "americanización en la economía española".

En 1954, el jefe del Servicio de Estadística en el Sindicato Vertical de Industrias Químicas, Ángel Rubio Carsi (1954), nos ofrecía un estudio cuantitativo de la industria química española a comienzos de los años cincuenta ${ }^{17}$, en su mayoría concentrada entre Barcelona, Madrid, Valencia y Vizcaya; las dos primeras provincias sumaban el $35 \%$ de las industrias y el $41,12 \%$ (el $30,61 \%$ sólo en Barcelona) de "la actividad económica nacional”. Los grupos incluidos en el estudio de Ángel Rubio eran los que, en aquella época, conformaban el Sindicato Vertical de Industrias Químicas, es decir, aceites industriales, insecticidas, colorantes, perfumería, alcoholes, resinas, caucho, explosivos, plásticos, colas, lubrificantes, ceras parafinadas, farmacéutica, destilación, orgánica, abonos, metaloides y ácidos; de ellos, el que contaba con un mayor número de establecimientos industriales era el de "farmacéutica", con 1.377 empresas, sobre un total de 8.730 industrias químicas. ${ }^{18}$ El dato de censo obrero para la industria química española era de 101.902 trabajadores, de los cuales 16.553 pertenecían a laboratorios farmacéuticos, el segundo sector con más obreros después del de ácidos. En cuanto a los capitales, el 11,50\% estaba en la industria farmacéutica, sólo superada por la industria de ácidos; 509.202.258 pesetas para un total de 4.425.031.069 en toda la industria química.

Como podemos apreciar, la industria farmacéutica ocupaba un lugar destacado en el sector químico; en palabras de Ángel Rubio Carsi (1954, p. 386), “(...) se aprecia (...) una manifiesta superioridad de la industria química farmacéutica, que engloba un máximo de concentración en establecimientos y capitales y también casi un máximo en los trabajadores que se dedican a esta actividad". El autor elabora una tabla de "importancia económica", valiéndose de tres tipos de porcentajes: capitales, número de trabajadores y número de establecimientos; en esa tabla figura a la cabeza el sector farmacéutico, con un valor en porcentaje de $20,50 \%$, seguido de la industria de ácidos con un 13,95\%; el resto de grupos bajan del $10 \%$. Sin embargo, las empresas españolas químico-farmacéuticas eran más pequeñas que las de otros sectores; según los datos manejados por Ángel Rubio Carsi, el número medio de empleados por laboratorio farmacéutico era de doce, superado por siete de los diecisiete grupos químicos del Sindicato, y el capital medio era de 369.790,8 pesetas, superado por doce de estos grupos.

Finalizado el período autárquico por excelencia (1939-1959) y según datos del Sindicato Vertical de Industrias Químicas del año $1961^{19}$, la industria farmacéutica se hallaba en segundo lugar en orden de importancia con respecto a todo el sector químico, sobrepasada ligeramente por la industria del caucho. Había en España un total de 536.288 establecimientos industriales; de ellos, 6.768 eran industrias químicas, entre las que se incluían a 756 laboratorios farmacéuticos (el $43,31 \%$ estaban en Barcelona y el $21,82 \%$ en Madrid). El total de empleados en la industria española se elevaba a 3.139.612 de "productores"; 109.547 trabajaban en el sector químico y aproximadamente una quinta parte estaba en laboratorios farmacéuti- 
$\cos (21.024 \text { trabajadores })^{20}$; en Madrid trabajaba el $44,52 \%$ del total de trabajadores y en Barcelona el $36,31 \%$. La media de empleados por planta para el total de la industria era de 5,9; 16,2 para la industria química y 27,8 para la farmacéutica, aunque estos datos no incluían a los laboratorios más pequeños, los anejos, categoría suprimida por la Ley de Bases de Sanidad Nacional de $1944 .^{21}$

\section{FACTORES A CORREGIR PARA EL ESTABLECIMIENTO DE UNA INDUSTRIA FARMACÉUTICA ESPAÑOLA AUTOSUFICIENTE}

Como hemos podido ver, finalizada la Autarquía el sector farmacéutico seguía ocupando un lugar destacado en el conglomerado de la química industrial, sin embargo eran empresas pequeñas, normalmente laboratorios de especialidades farmacéuticas en los que la química no solía estar presente. Albert Carreras y Xavier Tafunell (1993), en su documentado trabajo sobre las grandes empresas españolas del período 1917-1974, casi nunca mencionan a las industrias farmacéuticas entre las doscientas mayores empresas españolas ("clasificadas por activos netos"); de hecho, no aparece ninguna en las tablas correspondientes a los años 1917, 1930 y 1948; y, en 1960, tan solo figuran dos: Foret, en el puesto 172, y Antibióticos, S.A., en el puesto 185 .

El médico Antonio Gallego (1959) ${ }^{22}$, una de las figuras más destacadas de la histo-fisiología española durante el franquismo, distinguía entre dos tipos de laboratorios: los de "simple acabado de productos farmacéuticos" y "los fabricantes propiamente dichos". ${ }^{23}$ Los primeros no eran más que una extensión de la rebotica, donde se hacían fórmulas magistrales a gran escala a partir de materias primas que no producían, y siempre estaban supeditados y limitados por la política marcada por los productores de estas sustancias. En su opinión, este modelo de laboratorio farmacéutico español era dañino para nuestra industria; además de limitar el desarrollo global del sector, porque daba prioridad a este tipo de laboratorios frente a los químico-farmacéuticos, tendía a copiar los descubrimientos de otros, aumentaba innecesariamente la oferta de productos farmacéuticos y favorecía los contubernios $y$, en general, los comportamientos poco éticos. De esta misma opinión era José Amargós Anoro (1958) ${ }^{24}$, pensaba que la gran industria farmacéutica española aún estaba por construir, a pesar de las "notables y recientes realizaciones".

De algún modo, tanto Antonio Gallego como José Amargós criticaban los resultados de las políticas au- tárquicas en el ámbito farmacéutico, aunque bien es cierto que lo hicieron a finales de los años cincuenta, cuando este modelo económico ya estaba siendo superado. Sin embargo, estas voces discordantes ya se escuchaban desde 1942; en opinión de Emilio Plana Eichberg (1942), la Autarquía era difícil de lograr en el ámbito de la Química "si no se cuenta con un desarrollo suficiente", tal y como había hecho Alemania; pensaba que nos habíamos conformado con imitar a Francia" donde no había que recurrir al mercado de importación en busca de las materias necesarias para su composición". Unos años después, el farmacéutico Carlos Alfageme Rubio (1950) abundaba en esta opinión, sobre todo porque pensaba que el sector de las especialidades farmacéuticas ya estaba consolidado y en condiciones de abastecer el mercado nacional.

En el epígrafe anterior aludíamos a la cifra dada por el Sindicato Vertical de Industrias Químicas, en 1961, de 756 laboratorios farmacéuticos existentes en España. Sin embargo, el número de empresas tuvo que ser mayor, mas que nada porque los laboratorios anejos quedaban fuera de esta estadística. Lo cierto es que, entre 1939 y 1959, se encuadraron 1.121 laboratorios de especialidades farmacéuticas en el Sindicato Vertical de Industrias Químicas, de los cuales aproximadamente un 38\% eran anejos (Rodríguez Nozal, 2013). ${ }^{25}$

En 1958, el que fuera Jefe del Registro Farmacéutico de la Dirección General de Sanidad durante treinta años y Profesor del Laboratorio Municipal de Madrid, Antonio Serrada (1958), estimaba en unos 2.000 los laboratorios farmacéuticos asentados en España, una cifra excesiva desde su punto de vista; opinaba que, para que todo funcionara mejor, sólo debería haber "catorce o dieciséis [laboratorios] pero de gran envergadura", además, de esta manera se evitaría que muchos farmacéuticos tuvieran que tirar a la basura los medicamentos industriales cuando éstos caducaban o no se vendían; en su opinión, "con unos Laboratorios serios y formales se evitaría".

Por su parte, Antonio Gallego (1959, p. 29) cifraba en 1.800 los establecimientos farmacéuticos españoles, "muchos más que en los Estados Unidos", pero se preguntaba si, en realidad, eran "propiamente laboratorios como entidades científicas y económicas". En su opinión, urgía crear una industria química de base que atenuase nuestra dependencia del extranjero y que acabase, de una vez por todas, con los problemas en el suministro de materias primas y redujera los costes de producción, en definitiva "un plan de desarrollo para la industria farmacéutica que anulara su actual atomización, su desunión, ese ir cada uno por su lado". 
Como venimos comentando, la consecuencia inmediata de nuestro modelo productivo, de corte mediterráneo, fue una masificación poco operativa en la oferta de medicamentos industriales, algo que es consecuencia directa de la incorporación del profesional de la Farmacia a la producción a gran escala; así, un mismo medicamento era comercializado, bajo nombres y marcas diferentes, en función del farmacéutico fabricante. En definitiva, copias de copias que, a su vez, copiaban los originales, generalmente procedentes del extranjero. A menudo eran medicamentos creados con un único fin, el de ser incluidos en las listas del Seguro Obligatorio de Enfermedad (Redondo Rincón, 2012). Una manera de entender la industria farmacéutica muy criticada por Antonio Gallego (1959, p. 28), por oportunista y regirse por un "deseo de grandes beneficios en el menor tiempo posible (...) En vez de responder a una necesidad de consumo". Con este mecanismo los farmacéuticos aseguraban sus ingresos y, por lo tanto, la viabilidad empresarial de estos pequeños negocios, bien alejados de la creatividad y la innovación de las grandes empresas farmacéuticas del sector.

En cuanto a los productos fabricados por nuestra industria, algunos farmacéuticos ([Ion], 1942, p. 10) calculaban que, en 1936 , el $70 \%$ de las especialidades farmacéuticas "consumidas en nuestro mercado interior eran de origen extranjero"; quizás por ello, durante la Guerra Civil, las de fabricación nacional resurgieron, "comenzando a sustituir paulatinamente a las extranjeras y en un buen número de casos eclipsándolas (...) hasta el punto de que muchas de ellas se han impuesto, adoptándose por la clase médica sin reparos ni temores". Sin embargo, esta irrupción del producto hispano se realizó sin controles adecuados, lo que acabaría provocando "una situación de anarquía absoluta, que fue aprovechada por algunos desaprensivos con la vista puesta en su exclusivo beneficio comercial" ([Farmacia Nueva], 1940).

Según datos proporcionados por Fidel-Enrique Raurich Sas (1945, pp. 8-9), Catedrático de Farmacia y "Técnico excedente del actual Centro Técnico de Farmacobiología", el número de especialidades farmacéuticas registradas, a 31-XII-1939, Ilegaba a las 22.000 en números redondos, y eso que una quinta parte fueron rechazadas tras los análisis efectuados por el Instituto Técnico de Comprobación ${ }^{26}$, desde su creación el 15-VII-1927 hasta el 31-XII-1937.

En 1951, Javier Blanco Juste (1951) hablaba de unas 6.000 especialidades "en vigencia" y unas 46.000 "en el panteón del olvido" y no dudaba calificar a la sección de Registros de la Inspección General de Farmacia, como de "archivo copioso", con "toneladas de papel" y "tramitación rigurosa", una "verdadera colmena de trabajo". Entendía que la especialidad farmacéutica, en 1951, no era un negocio próspero para los laboratorios ni un producto especialmente atractivo para las oficinas de farmacia, los pacientes, los médicos o los almacenistas. ${ }^{27}$

En definitiva, el excesivo número de laboratorios farmacéuticos y de productos comercializados actuaba como freno para el desarrollo de la gran industria químico-farmacéutica; incluso el propio Director General de Sanidad, el farmacéutico Nazario Díaz, pensaba que, "en interés de la salud pública y de los farmacéuticos españoles", lo ideal sería disminuir a la mitad el número de medicamentos industriales comercializados; aunque también era consciente del fuerte arraigo de esta manera de entender la industria farmacéutica en España, por lo que tampoco consideraba buena idea el hecho de iniciar una "ofensiva bélica con esta intención" (Volpone, 1950).

Otro de los factores que, tal vez, pudieron ralentizar el desarrollo de la industria químico-farmacéutica fue el de los capitales, quizás más necesarios en empresas grandes, generalmente organizadas como sociedades anónimas y con una diferenciación clara entre el personal técnico y el núcleo capitalista; justo lo contrario que sucedía en nuestro país, donde la industria del medicamento se concebía como extensión de la rebotica y, en el mejor de los casos, como sociedades colectivas de ámbito familiar (Rodríguez Nozal y González Bueno, 2005; Rodríguez Nozal y González Bueno, 2008). La propia concepción autárquica de la economía española durante los años cuarenta y cincuenta dificultaba la entrada de capitales extranjeros, aunque ya hemos comentado anteriormente que esto no fue del todo así; a comienzos de los años cuarenta la presencia alemana seguía siendo importante y, a partir de los años cincuenta, el protagonismo inversor estadounidense fue algo evidente. En cambio, durante los años cuarenta no hubo muchas iniciativas de concentración inversora entre el fragmentado colectivo empresarial de la industria farmacéutica; en este sentido, los proyectos más importantes fueron los de CEPA y Antibióticos, S.A., ambos en torno a la penicilina. A comienzos de los sesenta aún se seguía abogando por la concentración empresarial (Abelló Pascual; Mayor Domingo y Donada Bosch, 1963) y, en 1972, ya se había logrado disminuir el número de laboratorios hasta los 507, desde los aproximadamente 1.200 que había en 1956 ó los 639 de 1967 (Donoso, 1978). 
Pero la industria químico-farmacéutica, además de capitales y de un cierto grado de concentración empresarial, necesitaba de personal cualificado en ámbitos como la Microbiología, la Ingeniería Química - la Química Orgánica. Ya en 1944, desde la revista Farmacia Nueva (1944) se pensaba que "la industria química española, incluida la farmacéutica, necesita de una manera apremiante de técnicos capaces, efectivamente capaces, y ellos han de salir, después de la ordenación de las Facultades, de una excelente cantera: la universitaria". El colectivo farmacéutico consideraba que la carrera de Farmacia era una de las más indicadas para liderar este giro hacia lo industrial pues, como manifestaban algunos de sus profesionales, "a nosotros toca continuar dando impulso a nuestra profesión, en sus diversas facetas, hacia una mayor grandeza" (Ajuria, 1944). Y eso a pesar de que, en 1944, se había establecido un doctorado universitario en Química Industrial dirigido exclusivamente a los titulados en Ciencias $^{28}$. Un asunto que no gustó mucho a algunos farmacéuticos por considerar injusto que se les excluyera de esta posibilidad formativa; la línea editorial de Farmacia Nueva (1945) defendía que gran parte de las industrias químicas españolas habían sido (y continuaban siendo) dirigidas por farmacéuticos; además, recordaban que una parte importante de la industria química era químico-farmacéutica y, finalmente, porque la preparación química de los farmacéuticos no era inferior a la de los químicos titulados.

Pocos años después, Carlos Alfageme Rubio (1950), en el marco del I Congreso Hispano-Portugués de Farmacia, realizaba una propuesta de lo que, a su juicio, debería ser la formación del farmacéutico en materia de química industrial. Ésta pasaba por "ir considerando dentro de cada asignatura el aspecto industrial de la misma"; plantear visitas a fábricas y laboratorios, "lo que contribuiría a completar la preparación de los Farmacéuticos y a despertar tempranas vocaciones por la Industria tan prometedora y que tan vastas posibilidades ofrece a nuestra Clase"; incluir una mayor formación en química farmacéutica de tipo técnico industrial, incluso con alguna asignatura nueva; y la posibilidad de realizar tesis doctorales en esta materia. De esta manera, pensaba que se podría tener un farmacéutico "perfectamente capacitado para desempeñar desde el primer día las misiones que se le encomienden dentro del campo industrial".

Finalizando la Autarquía, Antonio Gallego (1959) seguía pensando que la industria farmacéutica española no estaba técnicamente capacitada y que esa era una de las razones por las que se limitaba a copiar lo que proponían en el extranjero y a abrir laboratorios de acabado. Para él, la culpa de esta situación no había que buscarla únicamente en la insuficiente formación químico-industrial industrial recibida en las aulas, también hacía responsable a la política industrial española en esta materia y a las propias empresas, incapaces de formar a equipos de técnicos, científicos e investigadores suficientemente capacitados. Una vez más, en opinión de este médico, había que mirar a los países más desarrollados, donde se gastaba entre el 7 y el $15 \%$ de sus ingresos brutos en investigación científica; con todo, admitía que algunos laboratorios ya estaban empezando a gastar dinero en este apartado y que habían iniciado contactos con centros de investigación oficiales, sobre todo universitarios. En su opinión, "la industria debe darse cuenta de que su porvenir depende de aquello que pueda producir con carácter auténticamente original" ${ }^{29}$, planteamiento que aún tiene valor y que, de algún modo, trata de ser asumido por una industria farmacéutica cada vez más concentrada en unos pocos grupos empresariales de carácter multinacional y, en lo que respecta a nuestro país, ya alejada del viejo modelo mediterráneo de fragmentación industrial, caracterizado por el predominio de la empresa familiar y la simplicidad de los planteamientos científico-técnicos.

\section{CONCLUSIÓN}

La industria de las materias primas para la producción de medicamentos apenas fue desarrollada en España con anterioridad a la Guerra Civil. La implantación de las políticas autárquicas, características del primer franquismo, posibilitaron un cierto interés de nuestros gobernantes hacia este tipo de actividades fabriles; en primer lugar, tal y como ya se venía haciendo antes de 1936, promocionando industrias de productos naturales con el objetivo de obtener principios activos de acción medicinal; en segundo lugar, a más largo plazo, tratando de crear en España una industria química de base orgánica, que permitiera sintetizar los productos que habitualmente se adquirían en el extranjero; por último, haciendo todo lo posible por incorporarse al incipiente mercado de los antibióticos.

En cuanto a los dos primeros objetivos (productos naturales y materias primas de origen químico), los resultados no fueron muy alentadores y, en absoluto, se cumplieron los ideales perseguidos de autarquía económica; se continuaron exportando plantas medicinales, que volvían a entrar en España transformadas en principios activos, mientras que la industria quími- 
co-farmacéutica apenas avanzó durante estos años, algo reconocido incluso por quienes, desde la utopía y la propaganda franquista, entendían que aún se necesitaban medios y tiempo para establecer programas industriales de este calado.

En el terreno de los productos biológicos hubo más avances, aunque también es cierto que es un ámbito en el que se hicieron muchos progresos durante el primer tercio del siglo XX; de hecho, buena parte de los laboratorios productores de sueros y vacunas acabarían conformando el núcleo accionarial de Antibióticos, S.A., uno de los dos consorcios que se repartieron el mercado de los antibióticos en España a finales de los años cuarenta. La producción autóctona de antibióticos fue la gran apuesta del primer franquismo en materia químico-farmacéutica, aunque tampoco fue un proyecto totalmente autárquico, ya que necesitó de acuerdos científicos y tecnológicos con los Estados Unidos; esta colaboración se vio favorecida por la adhesión de España a los acuerdos de Bretton Woods y por el fenómeno conocido como "americanización en la economía española", especialmente notorio en los años sesenta, aunque en la década anterior ya había empezado a evidenciarse; en este sentido, la participación americana en el programa nacional de producción de antibióticos,

\section{NOTAS}

* Financiado con cargo al proyecto de investigación HAR201342536-P del Ministerio de Economía y Competitividad.

1. Sobre la tipificación de los modelos "centroeuropeo" y "mediterráneo" para la industria farmacéutica, véase González Bueno y Rodríguez Nozal (2010); Rodríguez Nozal y González Bueno (2008).

2. El Anuario de Industrias Químicas recoge cinco empresas de colorantes artificiales establecidas en España, todas ellas en Barcelona: Fabricación Nacional de Colorantes y Explosivos, Preckler y Alemany, Industrias y Manufacturas, S.A., Cardoner y Cía y la Sociedad Anónima Cros (Peres Unzueta, 1932, pp. 67-70).

3. Un tabla con la mayor parte de los fabricantes españoles de productos químicos de utilización en terapéutica, realizada a partir de los datos del Anuario de Industrias Químicas, una publicación, editada por la organización que englobaba a la mayoría de empresas del sector -la Cámara Nacional de Industrias Químicas-, puede consultarse en Rodríguez Nozal y González Bueno (2005, pp. 184-192). Un estudio sobre la industrialización de las algas marinas en la España anterior a la Guerra Civil, en el trabajo de Dosil Mancilla (2007).

4. Vocal de la Junta Nacional de Farmacia gestada en noviembre de 1936, presidente del Comité Sindical de In- iniciado en 1949, bien podría considerarse como un antecedente de este proceso de americanización de nuestra economía.

Trabajos como el de Albert Carreras y Xavier Tafunell (1993) no mencionan a las industrias farmacéuticas españolas entre las 200 mayores empresas españolas ("clasificadas por activos netos"), al menos en sus tablas elaboradas para los años 1917, 1930 y 1948. Sin embargo, según datos proporcionados por el Sindicato Vertical de Industrias Químicas, la industria farmacéutica ocupaba un lugar destacado en el conjunto de las industrias químicas -sobre todo en lo relativo a número de laboratorios-, aunque es verdad que éstos eran pequeños en capitalización y en número de empleados; además, los desarrollos científicos, sobre todo los de tipo químico, eran casi inexistentes. Todos estos factores contribuyeron a frenar el desarrollo de la industria farmacéutica española durante la Autarquía; aunque también hubo otros condicionantes, como las propias limitaciones de las políticas autárquicas, la escasez de materias primas, la insuficiente capacitación científico-técnica necesaria para esta actividad o la adscripción de nuestra industria a un modelo mediterráneo, tendente a capitalizaciones de índole intraprofesional o familiar y orientado hacia los desarrollos galénicos en lugar de los químicos.

dustrias Químico-farmacéuticas desde 1938 y, desde 1939, máximo responsable de las ramas de "especialidades farmacéuticas", productos galénicos y su síntesis, productos dietéticos y organoterapia de la Comisión Reguladora de las Industrias Químicas; hasta su jubilación, estuvo vinculado a la estructura administrativa del Ministerio de Industria y Comercio, y durante mucho tiempo fue Secretario General del Sindicato Vertical de Industrias Químicas y vocal del Instituto Técnico de Farmacobiología; fue miembro de la Real Academia Nacional de Farmacia. Más información sobre este autor en: Volpone (1940); Roldán Guerrero (1958-1963, 4: 714-715); Reol Tejada (1988); Vián Ortuño (1988); García Ortiz (1988); González Bueno (2010); Castillo Rodríguez (2011); González Bueno (2013b); Puerto Sarmiento (2012).

5. Su primer presidente fue el farmacéutico Santiago Pagés Maruny (Rodríguez Nozal, 2011a).

6. En 1941 se llegó a legislar sobre este particular, en el sentido de impedir que los funcionarios pudieran tener ligazones con empresas privadas ([Farmacia Nueva], 1941).

7. Sobre los antecedentes del Sindicato Vertical de Industrias Químicas y el papel desempeñado por este organismo en el control de cupos, véase Rodríguez Nozal (2013). 
8. Ambas empresas estuvieron vinculadas a grupos empresariales favorecidos por el franquismo; FAES a la familia Basagoiti y al Banco Hispano-Americano (Fernández Pérez, 2013; Andrés Turrión, 2013), mientras que al laboratorio Abelló no le debió resultar muy difícil subsistir en plena escasez de materias primas, no en vano Fernando Abelló Pascual llegó a ser el Vicepresidente del Sindicato Vertical de Industrias Químicas (Chaqués Bonafont, 2000), mientras que Juan Abelló Pascual prestó servicio en el Ejército afecto a Francisco Franco; en 1939 fue nombrado Presidente de la Rama de reactivos, Rama de las porcelanas y vidrios químicos y Rama del material del laboratorio, dependientes de la Comisión Reguladora de las Industrias Químicas (González Bueno, 2013a). Como comentaremos más adelante, estos dos laboratorios formaron parte de los dos grupos favorecidos por el gobierno de Franco para la fabricación de antibióticos en nuestro país; FAES se integró en CEPA, mientras que Abelló lo hizo en Antibióticos, S.A.

9. Se editó, de manera independiente, como folleto de 43 páginas (Villanueva Vadillo, 1948). También fue publicado por la revista profesional Farmacia Nueva, 13(137): 301310. 1948; e incluido en las actas del / Congreso HispanoPortugués de Farmacia. Madrid - Ciudad Universitaria, 30 de mayo a 6 de junio, 1948: 725-742 (Madrid: Real Academia de Farmacia, 1950). Fue reseñado, de manera anónima, bajo el título de "Progreso de la industria farmacéutica nacional en el último decenio. Interesante trabajo del Doctor Villanueva Vadillo", por Información Comercial Española, [1948] (agosto): 40-41. 1948.

10. Una información más detallada sobre estos productos químico orgánicos fabricados en España, en el trabajo de Villanueva Vadillo (1948).

11. En este mismo sentido, eufórico y propagandístico, se manifestaban Eliseo Bermudo Soriano (1952, p. 1758), quien llega a afirmar "que los productos españoles [de tipo farmacéutico] se imponen hoy en el mercado internacional"; y, por supuesto, Villanueva Vadillo (1951).

12. Una información más extensa sobre este asunto en Santesmases (1999); Santesmases (2010); Rodríguez Nozal (2011a); Santesmases y Gradman (2011); Romero de Pablos (2011), González Bueno; Rodríguez Nozal y Pérez Teijón (2012); González Bueno y Rodríguez Nozal (2012).

13. Sobre el grupo farmacéutico Alter véase Rodríguez Nozal y González Bueno (2015a); Rodríguez Nozal y González Bueno (2015b).

14. De la Leo-Penicilina se ha ocupado Cozzoli (2014).

15. Sobre el proceso de expropiación de las empresas alemanas en España a partir de 1945, véase el trabajo de Puig Raposo y Álvaro Moya (2007).

16. De las alianzas económicas entre España y Estados Unidos durante los años cincuenta y sesenta se han ocupado Puig Raposo y Álvaro Moya (2004).
17. Un catálogo donde se recogen todos los productos químicos españoles, por orden alfabético, junto con el nombre del fabricante, la ciudad y el domicilio del mismo, así como la "clase", es decir, si eran reactivos para análisis, productos industriales o farmacéuticos en: [Anónimo] (1955).

18. El Anuario de la Industria Química Española de 1953, editado por el Sindicato Vertical de Industrias Químicas, contabiliza 7.500 empresas químicas con 11.500 fábricas; de ellas, más o menos 450 eran grandes, 2.500 medianas y el resto pequeñas o artesanales (consultado en Diego García, 1996, p. 83).

19. Consultados en el trabajo de Abelló Pascual; Mayor Domingo y Donada Bosch (1963). También de interés el texto de Brosa Rabassa (1963). Francisco Abelló Pascual era hermano de Juan Abelló (véase la nota 8); Federico Mayor Domingo fue gerente del Laboratorio Leti-Uquifa, miembro del primer Consejo de Administración de Antibióticos, S.A. y presidente de la Comisión Asesora de Investigación Científica y Técnica adscrita al Ministerio de la Presidencia durante la Transición (Redondo Rincón y González Bueno, 2013; Lobo, 1979); Francisco Donada Bosch fue miembro de la Academia de Farmacia de Barcelona; y Salvador Brosa Rabassa trabajó como director técnico del Laboratorio Andreu y fue miembro fundador de la Real Academia de Farmacia de Cataluña (Roca i Torras, 1993: 12-14).

20. Algunas fuentes redondean a 18.000 trabajadores para unos 800 laboratorios farmacéuticos, lo que suponía un $15,2 \%$ del total productor en el sector químico (la industria del caucho llegaba al $15,8 \%$ ). El valor bruto de los medicamentos industriales obtenidos superó los seis mil millones de pesetas en 1961 (Pozo y Donada, 1962, p. 688). La estimación ofrecida por uno de los colaboradores de la revista Circular Farmacéutica, en cuanto a número de trabajadores se refiere, era parecida: un $15 \%$ del censo laboral de la industria química correspondía a los laboratorios farmacéuticos (Dr. D. Antonio, 1956).

21. En la Base decimosexta de esta disposición se podía leer: "Los laboratorios destinados a la preparación de especialidades farmacéuticas podrán ser de dos clases: laboratorios individuales y laboratorios colectivos" (Ley de 25 de noviembre de 1944 de Bases de Sanidad Nacional -BOE, 26-XI-).

22. Catedrático de Universidad, Vicerrector de Investigación durante la Transición, Director del Instituto de Farmacología Española, miembro de la Real Academia Nacional de Medicina y de otras academias y sociedades; fue el primer director técnico del Laboratorio de la Compañía Española de Penicilina y Antibióticos (CEPA). Sobre este autor, véase Rodríguez Nozal (2004a); Santesmases (2001).

23. En 1948, a propósito del I Congreso Hispano-Portugués de Farmacia, Carlos Alfageme Rubio (1950) dividía a las industrias farmacéuticas de la siguiente manera: industrias extractivas, de medicamentos químicos, de productos obtenidos por fermentación, de sueros, vacunas y semejantes, de productos dietéticos y de "formas farmacéuticas de los medicamentos". 
24. Profesor Auxiliar de Química Orgánica en la Universidad de Barcelona, miembro del Consejo de Administración de la Sociedad Hijos del Dr. Andreu, S.A. y director técnico de este laboratorio, miembro fundador de la Real Academia de Farmacia de Cataluña (Roldán Guerrero, 1958-1963, 1: 154).

25. Finalizando la Autarquía Víctor Villanueva Vadillo (1957) cifraba en 1.200 las industrias químico-farmacéuticas españolas existentes.

26. Organismo creado por Real Decreto de 22-XII-1925 (Gaceta de 25-XII); las bases para la organización y reglamentación de sus funciones y servicios fueran publicadas en la Real Orden de 9-VII-1927 (Gaceta 13-VII-1927).

\section{BIBLIOGRAFÍA}

Abelló Pascual, Francisco; Mayor Domingo, Federico y Donada Bosch, Francisco (1963), El desarrollo económico de la industria farmacéutica en los próximos años, [S.I., s.n.] [Ponencia presentada a la II Convención Bienal de la Industria Farmacéutica Española. Ejemplar mecanografiado conservado en la Cátedra de Historia de la Farmacia y Legislación Farmacéutica, Facultad de Farmacia, UCM, signatura: HIS-F-264].

Abollado Aribau, Carlos (1945), La industria química y la química industrial, Madrid, Asociación Nacional de Ingenieros Industriales.

Agell i Agell, Josep (1926) "La industria química en Cataluña", Química e Industria, 29, pp. 147-153.

Ajuria, C.R. (1944), "Estado actual de la Farmacia”, El Monitor de la Farmacia y de la Terapéutica, 1342, pp. 176.

Alfageme Rubio, Carlos (1950), “La Farmacia galénica y las Industrias farmacéuticas". En: I Congreso Hispano-Portugués de Farmacia. Madrid - Ciudad Universitaria, 30 de mayo a 6 de junio, 1948, Madrid, Real Academia de Farmacia, pp. 658-675.

Amargós Anoro, José (1958), Comentarios de actualidad sobre la industria químico-farmacéutica de síntesis, Barcelona, Real Academia de Farmacia de Barcelona.

Andrés Turrión, María Luisa de (2013), "Medicamentos, análisis e informes técnicos: el Cuerpo Militar de Farmacia en la estructura sanitaria del Ministerio del Ejército (1939-1945)". En: González Bueno, Antonio y Baratas Díaz, Alfredo (eds.) La tutela imperfecta. Biología y Farmacia en la España del primer franquismo, Madrid, CSIC, pp. 101-141.

[Anónimo] (1955), Productos Químicos Españoles, 12 ed., Madrid, C. Sáez.

Arco Blanco, Miguel Ángel (2006), “'Morir de hambre'. Autarquía, escasez y enfermedad en la España del primer franquismo", Pasado y Memoria. Revista de Historia Contemporánea, 5, pp. 241-258.
27. Esta situación era reconocida incluso por el todopoderoso Víctor Villanueva: "El crecimiento numérico de las especialidades farmacéuticas no puede en modo alguno beneficiar ni a los laboratorios preparadores ni a la farmacia, tan atormentada por el excesivo surtido, ni al médico, confundiéndolo, ni al público consumidor" (Volpone, 1946, p. 303).

28. Orden de la Dirección General de Enseñanza Universitaria de 4-X-1944 (BOE, 5-X). Sobre este asunto véase Toca (2006).

29. Antonio Gallego no era el único en percibir esta necesidad, desde Farmacia Nueva también se estaba en que toda la responsabilidad formativa no debería recaer sobre la Universidad, sino en las propias industrias, además de incrementar el contacto con el exterior ([Farmacia Nueva] 1959).

Barciela, Carlos (ed.) (2003), Autarquía y Mercado Negro. El fracaso económico del primer franquismo (1939-1959), Barcelona, Crítica.

Bermudo Soriano, Eliseo (1952), "La Química, base de la industria moderna", Información Comercial Española, [1952] (noviembre), pp. 1754-1759.

Blanco Juste, Javier (1951), "Historia de la especialidad farmacéutica española”, Farmacia Nueva, 16(174), pp. 351-354.

Brosa Rabassa, Salvador (1963), Un cuarto de siglo en la industria químico-farmacéutica, Barcelona, Real Academia de Farmacia.

Carreras, Albert y Tafunell, Xavier. (1993), "La gran empresa en España (1917-1974). Una primera aproximación", Revista de Historia Industrial, 3, pp. 127-175.

Castillo Rodríguez, Carlos del (2011), "Los inicios burgaleses de Víctor Villanueva Vadillo (1906-1996): el Laboratorio Thor". En: González Bueno, Antonio et al. (eds.). Homenaje al Prof. Dr. José Luis Valverde, Granada, SDUHFE / Universidad de Granada, pp. 563-575.

Catalán, Jordi (1989), "Autarquía y desarrollo de la industria de fábrica durante la segunda guerra mundial. Un enfoque comparativo". En: García Delgado, José Luis (ed.) El primer franquismo. España durante la segunda guerra mundial. $V$ Coloquio de Historia Contemporánea de España, dirigido por M. Muñón de Lara, Madrid, Siglo XXI, pp. 35-88.

Catalán, Jordi (2002), “Franquismo y autarquía, 1939-1959: enfoques de historia económica", Ayer, 46, pp. 263-283.

Chaqués Bonafont, Laura (2000), "Continuidad y cambio en la organización de intereses de la industria farmacéutica", Gestión y Análisis de Políticas Públicas, 17-18, pp. 55-69.

Colomer i Roma, Pere (2003), "La política de la piel. El sector curtidos en España (1940-1980)", Revista de Historia Industrial, 24, pp. 115-159. 
Cozzoli, Daniele (2014), “Penicillin and the European Response to Post-War American Hegemony: the Case of Leo-penicillin", History and Technology, 30 (1-2), pp. 83-103.

Deu, Esteve y Llonch, Montserrat (2013), “Autarquía y atraso tecnológico en la industria textil española, 1939-1959", Investigaciones de Historia Económica, 9, pp. 11-21.

Diego García, Emilio de (1996), Historia de la Industria en España. La Química, Madrid, Escuela de Organización Industrial.

Donoso, Vicente (1978), "La industria farmacéutica en España. Oligopolio y colonización", Revista de Fomento Social, 129, pp. 79-90; 130, pp. 187-201.

Dosil Mancilla, Francisco Javier (2007), Los albores de la botánica marina española (1814-1939), Madrid, Consejo Superior de Investigaciones Científicas.

Dr. D. Antonio (1956), "Nuestra Industria Farmacéutica”, Circular Farmacéutica, 14(151-152), [s.p.]

Farmacia Nueva (1940), "Comentarios", Farmacia Nueva, 40, p. 39.

Farmacia Nueva (1941), "Flecha en el blanco", Farmacia Nueva, 6 (54), p. 39.

Farmacia Nueva (1944), "Comentarios", Farmacia Nueva, 94, pp. 645-646.

Farmacia Nueva (1945), "El Doctorado en Química Industrial", Farmacia Nueva, 10 (106), pp. 619-621.

Farmacia Nueva (1959), "Hacia una industria farmacéutica técnicamente capacitada", Farmacia Nueva, 24 (264), pp. 23-24.

Fernández Navarrete, Donato (2005), "La política económica exterior del franquismo: del aislamiento a la apertura", Historia Contemporánea, 30, pp. 49-78.

Fernández Pérez, Paloma (2013), La profesionalización de las empresas familiares. Madrid et al., Lid.

Fernández Prieto, Lourenzo (2007), El apagón tecnológico del franquismo. Estado e innovación en la agricultura española del siglo XX, Valencia, Tirant Lo Blanch.

Fontana, Josep (2004), "La utopía franquista: la economía de Robinson Crusoe". Cuadernos de Historia del Derecho (2004, vol. extraordinario), pp. 97-103.

Fraile Balbín, Pedro (1991), Industrialización y grupos de presión. La economía política de la protección en España 19001950, Madrid, Alianza.

[Gallego, Antonio -entrevista-] (1959) "Declaraciones del Profesor don Antonio Gallego acerca del momento actual de la industria químicofarmacéutica española", Farmacia Nueva, 24 (264), pp. 27-30.
Gálvez Muñoz, L. y Comín Comín, F. (2003), “Multinacionales, Atraso Tecnológico y Marco Institucional. Las nacionalizaciones de empresas extranjeras durante la autarquía franquista", Cuadernos de Economía y Dirección de la Empresa, 17, pp. 139-179.

García Delgado, José Luis (1994), "La industrialización y el desarrollo económico de España durante el Franquismo". En: Nadal, Jordi; Carreras Albert; y Sudrià, Carles (comps.), La Economía española en el siglo XX. Una perspectiva histórica, Barcelona, Ariel, pp. 164-189.

García Ortiz, Alberto (1998), "In memoriam [Víctor Villanueva Vadillo]", Anales de la Real Academia de Farmacia, 64, pp. 559-561.

Gómez Mendoza, Antonio (1997), “El fracaso de la autarquía: la política económica española y la posguerra mundial (19451959)", Espacio, Tiempo y Forma, Serie V, Historia Contemporánea, 10, pp. 297-313.

Gomis, Alberto (2008), "El medicamento biológico: sueros y vacunas en España”. En: Rodríguez Nozal, Raúl y González Bueno, Antonio (coords.), El medicamento de fabricación industrial en la España contemporánea, Madrid, CERSA, pp. 225-256.

González Bueno, Antonio (2010), “La organización farmacéutica profesional durante los primeros años del franquismo: la Junta Nacional de Farmacia (1936-1937)", Schironia, 9, pp. 52-54.

González Bueno, Antonio (2013a), "Abelló Pascual, Juan”. En: Diccionario Biográfico Español, 1. Madrid, Real Academia de la Historia, pp. 189-190.

González Bueno, Antonio (2013b), "Villanueva Vadillo, Victor". En: Diccionario Biográfico Español, 50. Madrid, Real Academia de la Historia, pp. 138-139.

González Bueno, Antonio y Rodríguez Nozal, Raúl (2010), “Innovation vs. Tradition: the election of an european way toward pharmaceutical industrialisation, 12th-20th centuries", Anales de la Real Academia Nacional de Farmacia, 76(4), pp. 459-478.

González Bueno, Antonio y Rodríguez Nozal, Raúl (2012), “La penicilina en la España franquista: importación, intervención e industrialización", Eidon, 38 (diciembre), edición online -http://www.revistaeidon.es/archivo/revista-eidon-n-38/ humanidades/118147-la-penicilina-en-la-espana-franquistaimportacion-intervencion-e-industrializacion-, consultado el 01/02/2016.

González Bueno, Antonio; Rodríguez Nozal, Raúl y Pérez Teijón, Carlos José (2012), "La penicilina en España. Difusión, propiedad industrial y negocio, en clave autárquica (19441959)", Estudos do Século XX, 12, pp. 273-289.

González González, Manuel-Jesús (1979), La Economía política del franquismo (1940-1970). Dirigismo, mercado y planificación, Madrid, Tecnos. 
Ion (1942), "Industria Químico-Farmacéutica y Sanitaria”, Ion, 2(6), pp. 10-12.

Lobo, Félix (1979), "Política científica y desarrollo económico. 1959-1979", Información Comercial Española, 552, pp. 35-46.

López García, Santiago M. y Cebrián Villar, Mar (2015), "La dimensión internacional de las transformaciones científicas y tecnológicas". En: González Redondo, Francisco de Asís (coord.), Ciencia y Técnica entre la Paz y la Guerra. 1714, 1814, 1914, Madrid, SEHCYT, pp. 407-413.

Martínez Segarra, Rosa María (1987), “Repertorio bibliográfico del período autárquico español (1936-1951)", Revista de Derecho Político, 24, pp. 231-270.

Miranda Encarnación, José Antonio (1994), “La industria del calzado española en la posguerra: los efectos del intervencionismo sobre una industria de bienes de consumo", Revista de Historia Económica, 12(2), pp. 317-339.

Miranda Encarnación, José Antonio (2003), "El fracaso de la industrialización autárquica”. En: Barciela, Carlos (ed.), Autarquía y mercado negro. El fracaso económico del primer franquismo, 1939-1959, Barcelona, Crítica, pp. 95-121.

Mora López, Antonio (1951), "La industria de síntesis orgánica en España", Información Comercial Española, pp. 2016-2023.

Moreno Fonseret, Roque (1999), "El régimen y la sociedad. Grupos de presión y concreción de intereses", Ayer, 33 [E] Primer franquismo (1939-1959)], pp. 87-113.

Moreno Fonseret, Roque (2002), "Política e instituciones económicas en el nuevo estado", Pasado y Memoria, 1 [Instituciones y Sociedad en el franquismo], pp. 1-65.

Peres Unzueta, J. (dir) (1932), Anuario de Industrias Químicas, Barcelona, Cámara Nacional de Industrias Químicas.

Plana Eichberg, Emilio (1942), "Industria de medicamentos químicos", Ion, 2(6), pp. 47-48.

Pozo, A. del y Donada, Francisco (1962), "La industria farmacéutica en su aspecto técnico profesional”, Ion, 22, pp. 682 y 688-689.

Puerto Sarmiento, Francisco Javier (2012), Historia de la Real Academia Nacional de Farmacia, Madrid, Real Academia Nacional de Farmacia.

Puig Raposo, Nùria (1999), "El crecimiento asistido de la industria química en España: Fabricación Nacional de Colorantes y Explosivos, 1922-1965", Revista de Historia Industrial, 15, pp. 105-136.

Puig Raposo, Nùria (2002), “Una multinacional holandesa en España: la Seda de Barcelona, 1925-1991", Revista de Historia Industrial, 21, pp. 123-158.
Puig Raposo, Nùria (2003), Bayer, Cepsa, Repsol, Puig, Shering y La Seda. Constructores de la química española, Madrid, Lid.

Puig Raposo, Nùria (2004), "Redes empresariales de oportunidad en la España del siglo XX: el caso de la industria químicofarmacéutica", Historia Empresarial, 812, pp. 179-188.

Puig Raposo, Nùria y Álvaro Moya, Adoración (2004), "La Guerra Fría y los empresarios españoles: la articulación de los intereses económicos de Estados Unidos en España, 1950-1975", Revista de Historia Económica, 22(2), pp. 387-424.

Puig Raposo, Nùria y Álvaro Moya, Adoración (2007), “¿Misión imposible?: La expropiación de las empresas alemanas en España (1945-1975)", Investigaciones de Historia Económica, 7, pp. 101-130.

Raurich Sas, Fidel-Enrique (1945), Trece años con la especialidad farmacéutica, Barcelona, Real Academia de Medicina y Cirugía de Barcelona.

Redondo Rincón, Gloria (2012), El Seguro Obligatorio de Enfermedad en España: responsables técnicos y políticos de su implantación durante el franquismo, Madrid, Universidad Complutense. [Tesis Doctoral inédita].

Redondo Rincón, Gloria y González Bueno, Antonio (2013), “Penicilina para la España del primer franquismo (1944-1959". En: González Bueno, Antonio y Baratas Díaz, Alfredo (eds.), La Tutela Imperfecta. Biología y Farmacia en la España del primer franquismo, Madrid, CSIC, pp. 241-293.

Reol Tejada, Juan Manuel (1988), "In memoriam [Víctor Villanueva Vadillo]", Anales de la Real Academia de Farmacia, 64, pp. 551-558.

Roca i Torras, Jaume (1993), Historia de los medicamentos veterinarios en Catalunya y de sus laboratorios farmacéuticoveterinarios [Discurso leído por... en el acto de su recepción celebrado en la Reial Acadèmia de Farmàcia de Barcelona el día 13 de mayo de 1993], Barcelona, Reial Acadèmia de Farmàcia de Catalunya.

Rodríguez Nozal, Raúl (2004a), Uriach - Cambronero - Gallego. Farmacia e Industria. La producción de los primeros medicamentos en España, Madrid, Nivola.

Rodríguez Nozal, Raúl (2004b), “En torno al establecimiento de una industria químico-farmacéutica en España con anterioridad a la guerra civil". En: José Javier Escribano Benito et. al. (coord.), Actas del VIII Congreso de la SEHCYT. Historia de las Ciencias y de las Técnicas, Pamplona, SEHCYT / Universidad de la Rioja, vol. 2, pp. 739-755.

Rodríguez Nozal, Raúl (2011a), "La industria del medicamento en el seno del colectivo químico industrial: la Unión Nacional de Laboratorios Químico-Farmacéuticos (1919-1936)", Asclepio, 63(2), pp. 431-452. 
Rodríguez Nozal, Raúl (2011b), “¿Fortuna o desdicha? La entrada de la penicilina en la España de la autarquía, un nuevo desafío para la farmacia y la industria farmacéutica". En: González Bueno, Antonio et al. (ed.), Homenaje al Prof. Dr. José Luis Valverde, Granada, SDUHFE / Universidad de Granada, pp. 515-550.

Rodríguez Nozal, Raúl (2013), "La industria farmacéutica española durante la Autarquía. Estudio cuantitativo de los laboratorios registrados por la organización sindical". En: González Bueno, Antonio y Baratas Díaz, Alfredo (eds.), La tutela imperfecta. Biología y Farmacia en la España del primer franquismo, Madrid, CSIC, pp. 143-188.

Rodríguez Nozal, Raúl y González Bueno, Antonio (2005), Entre el arte y la técnica. Los orígenes de la fabricación industrial del medicamento, Madrid, CSIC.

Rodríguez Nozal, Raúl y González Bueno, Antonio (2008), “El modelo de industrialización farmacéutica española: aportes para una tipificación". En: Rodríguez Nozal, Raúl González Bueno, Antonio (coords.), El medicamento de fabricación industrial en la España contemporánea, Madrid: CERSA, pp. 5-12.

Rodríguez Nozal, Raúl y González Bueno, Antonio (2015a), "La doctrina social católica en el proceso industrializador de la España franquista: el caso del grupo farmacéutico Alter", Dynamis, 35(2), pp. 433-457.

Rodríguez Nozal, Raúl y González Bueno, Antonio (2015b), "Obreros penitentes: el programa social del grupo farmacéutico Alter durante el franquismo". En: Gomis Blanco, Alberto y Rodríguez Nozal, Raúl (eds.), De la Botica de El Escorial a la Industria farmacéutica: en torno al medicamento, Alcalá de Henares, Universidad de Alcalá, pp. 381-408.

Roldán Guerrero, Rafael (1958-1963), Diccionario biográfico y bibliográfico de autores farmacéuticos españoles, 4 vols., Madrid, IMPHOE.

Romero de Pablos, Ana (2011), "Regulation and the circulation of knowledge: Penicillin patents in Spain", Dynamis, 31(2), pp. 363-383.

Rubio Carsi, Ángel (1954), “Algunos aspectos de la situación y características de los establecimientos químicos españoles, Ion, 14, pp. 185-186; 14, pp. 254-255; 14, pp. 386-402.

Sánchez Recio, Glicerio (2003), "El franquismo como red de intereses". En: Sánchez Recio, Glicerio y Tascón Fernández, Julio (eds.), Los empresarios de Franco. Política y Economía en España, 1936-1957, Barcelona, Crítica-Universidad de Alicante, pp. 11-22.

Santesmases, María Jesús (1999), Antibióticos en la Autarquía: banca privada, industria farmacéutica, investigación científica y cultura liberal en España, 1940-1960, Madrid, Fundación Empresa Pública.

Santesmases, María Jesús (2001), Entre Cajal y Ochoa. Ciencias Biomédicas en la España de Franco, 1939-1975, Madrid, CSIC.
Santesmases, María Jesús (2010), “Distributing Penicillin: the clinic, the hero and industrial production in Spain. En: Quirke, Viviane y Slinn, Judy (eds.), Perspectives on Twentieth-Century Pharmaceuticals: 91-118. Oxford et al., Peter Lang, pp. 91-118.

Santesmases, María Jesús y Gradman, Christoph. (eds)- (2011), "Dossier: Circulation of Antibiotics: Historical Reconstructions", Dynamis, 31(2), pp. 293-427

[Serrada, Antonio -entrevista-] (1958), "El caso de las "especialidades" farmacéuticas". Farmacia Nueva, 23(259), pp. 391-392.

Tamames Gómez, Ramón (2005), "La Autarquía española y las rémoras para el crecimiento económico posterior", Información Comercial Española, 826, pp. 13-24.

Toca, Ángel (2006), “Dos profesiones para un solo cometido". La introducción de la ingeniería química en España durante el primer franquismo", Dynamis, 26, pp. 253-285.

Un Colaborador (1942) "La industria farmacéutica nacional", El Monitor de la Farmacia y de la Terapéutica, 1300, pp. 248-249.

Vián Ortuño, Ángel (1998), “En memoria de D. Víctor Villanueva Vadillo", Anales de la Real Academia de Farmacia, 64, pp. 543-550.

Villanueva Vadillo, Víctor (1945), Factores determinantes de un proyecto de industria químico-farmacéutica nacional, Madrid, Real Academia de Farmacia.

Villanueva Vadillo, Víctor (1948), "El progreso de la industria farmacéutica española en el último decenio", Farmacia Nueva, 13 (137), pp. 301-310.

Villanueva Vadillo, Víctor (1951), "Situación y perspectivas de la industria química española", Información Comercial Española, pp. 2000-2003.

Villanueva Vadillo, Víctor (1952), "Situación y perspectivas de la industria química española”, Ion, 12 (127), pp. 56-58.

Villanueva Vadillo, Víctor. (1957), "La industria químicofarmacéutica en España.- Su desarrollo desde el año 1939 y su influencia en la economía y en la sanidad", El Monitor de la Farmacia y de la Terapéutica, 1661, pp. 318-322.

Villanueva Vadillo, Víctor; Chocano, Vicente y Gil Collado, Juan (1955), "Industria farmacéutica e insecticidas", Ion, 15(172), pp. 618-624.

Volpone [Busto de Medrano, Pío del] (1940), "Víctor Villanueva", Farmacia Nueva, 5 (37), pp. 9-11.

Volpone [Busto de Medrano, Pío del] (1946), "Un reportaje de Volpone. "Volpone" se entrevista con D. Víctor Villanueva, Secretario Nacional del Sindicato Vertical de Industrias Químicas", Farmacia Nueva, 11(113), pp. 298-303.

Volpone [Busto de Medrano, Pío del] (1950), “Don Nazario Díaz expone su opinión sobre el registro de las Especialidades Farmacéuticas", Farmacia Nueva, 15(167), pp. 525-528. 